\title{
The dynamic field in turbulent round jet discharging into a co-flowing stream
}

\author{
Mohamed Hichem Gazzah $^{{ }^{*}}$, Nejmiddin Boughattas ${ }^{2}$, Hafedh Belmabrouk ${ }^{1}$, Rachid Said ${ }^{2}$ \\ ${ }^{1}$ Département de Physique, Faculté des Sciences de Monastir, Monastir, Tunisie; *Corresponding Author: \\ Hichem.Gazzah@,fsm.rnu.tn \\ ${ }^{2}$ UREMIR, Institut Préparatoire aux Etudes d'Ingénieurs de Monastir, Monastir, Tunisie
}

Received 22 February 2010; revised 16 March 2010; accepted 7 April 2010.

\begin{abstract}
The effects of a co-flow on a spreading and entrainment rate of turbulent round jets have been studied numerically. The first and second order closure models are used and have been compared with existing experimental data. The influence of theses models on the dynamic fields is examined. The results of the models in general agree well with the trends observed experimenttally. The co-flowing imposed noticeable restrictions on the spreading and the turbulent mixing. Finally, an entrainment hypothesis has been introduced to describe the development of turbulent jets issuing into a stagnant or co-flowing air. It relates the mass flow rate of the surrounding fluid entrained into the jet to the characteristic velocity difference between the jet and the co-flow. It is obvious that the co-flow decreases considerably the entrainment of air.
\end{abstract}

Keywords: Co-Flow; Turbulence; Jets; Models; Entrainment

\section{INTRODUCTION}

The experimental and numerical studies concerning the aerodynamics of co-flowing turbulent round jets have lately observed an increasing interest as they are encountered in several industrial applications, such as turbulent diffusion flames of combustion chambers where a fuel jet flow is commonly injected into a co-flowing stream jet. The important parameters that influence the mixing characteristics of a jet are the presence of density difference and a co-flowing between the jet and its surroundings.

The effect of the density variation in the turbulent round free jet has been investigated experimentally and numerically by several authors like [1-6]. The results of these works show that the mean and turbulent quantities are strong functions of density ratio. This confirms a higher mixing efficiency when the density ratio between the jet and the quiescent air decreases. It is shown that the density effects are affected by the buoyancy terms in the similarity region of the jet. The influence of the emission initial conditions on the evolution of the dynamic and scalar fields is also studied.

The present study is a continuation of the work started by the authors $[7,8]$ to investigate numerically the influence of the co-flow surrounding the turbulent jets. In this numerical study, several traditional scalar dissipation rate models are examined for scalar transport modeling in mixing turbulent round jets with co-flowing air. Therefore, it is not intended here to review previous work detail [9-11] on a co-flowing jet, since a review on the subject matter is given by the references $[7,8]$. The experimental and numerical investigations of this type of flow are also relatively scarce.

In his recent work, Hakem et al. [12], have studied experimentally the mixing characteristics of an elliptical jet with large varying aspect ratio in a co-flow current, to verify that the Elliptic jet with varying large aspect ratio has also much higher dilution in a co-flow than an equivalent round jet under the same flow conditions. Wang et al. [13] have also studied the variable-density turbulent round jets discharging into a weakly confined low-speed co-flowing air stream with the aid of largeeddy simulation. Thus, the majority of this work shows that the effects of the co-flow variation on the structure of the reactive and non-reactive turbulent jets are complex problems and remain very interesting.

The study of the jets with co-flow shows the presence of three essential areas: an initial area, a principal area and an area of transition. When the flow is confined, the process of the co-flow driven by the jet is modified and the mixing process depends strongly not only on the velocity ratio, but also on the interaction between the boundary layer, the mixing layer and the main flow. 
A considerable pressure gradient can appear and generates phenomena of recirculation. Curtet [14] is interested in a parameter of similarity, called parameter of Craya-Curtet which is formulated in the literature in variable density by Steward and Gurus [15]. He showed that, for a value of this number higher than 0.8 , the phenomenon of recirculation is avoided, irrespective of the fluid considered.

The major objective of this paper is to determine the effect of a co-flowing on the dynamic fields of a turbulent round jet. Therefore, we investigate a turbulent round jet into a co-flowing air with various co-flow to fuel velocity ratios. We have thus used first and second order closure methods to investigate and compare their performances. The influence of the co-flow on various physical parameters of the jet is analyzed in comparison with the experimental data of Djeridane [16].

\section{TURBULENCE MODELS}

The equations which govern the turbulent flow are derived from the conservation laws of mass and momentum. All variables are conventionally averaged. These conventional averaged variables are denoted by an overbar $\bar{\Phi}$. Conventional fluctuations are indicated by $\Phi^{\prime}$.

$$
\Phi=\bar{\Phi}+\Phi^{\prime}
$$

\subsection{The Mean Equations}

We suppose that, the mean motion is steady, the turbulent Reynolds numbers are high enough and the molecular diffusion effects are neglected.

The continuity equation is given by

$$
\rho \frac{\partial \bar{U}_{j}}{\partial x_{j}}=0
$$

The momentum equation is:

$$
\rho \frac{\partial}{\partial x_{j}}\left(\bar{U}_{i} \bar{U}_{j}\right)=-\frac{\partial \bar{p}}{\partial x_{i}}-\rho \frac{\partial}{\partial x_{j}}\left(\overline{u_{i}^{\prime} u_{j}^{\prime}}\right)+\rho g_{i}
$$

As a consequence of the nonlinearity (2), the averaging process used introduces unknown correlations which are modelled through turbulence models. In order to solve transport equation for mean velocity in the turbulent jets, the turbulent Reynolds stress shown in these equations is computed using two turbulence closure models, called the $k-\varepsilon$ model and the second order model. Details of theses models can be found in Schiestel [17].

\subsection{The First-Order $k-\varepsilon$ Model}

The turbulent fluxes are approximated with $k$ - $\varepsilon$ model. The Reynolds stresses tensors are related to the strain rate by the following equation:

$$
\overline{\rho u_{i}^{\prime} u_{j}^{\prime}}=-\mu_{t}\left(\frac{\partial \bar{U}_{i}}{\partial x_{j}}+\frac{\partial \bar{U}_{j}}{\partial x_{i}}\right)+\frac{2}{3} \bar{\rho} k \delta_{i j}
$$

where $\mu_{t}$ is the turbulent viscosity, which is obtained from the turbulent kinetic energy $k$ and its dissipation rate $\varepsilon$ using the relation:

$$
\mu_{t}=\rho C_{\mu} \frac{k^{2}}{\varepsilon}
$$

The turbulence model consists of equations for the turbulent kinetic energy and their dissipation. These are

The kinetic energy equation

$$
\rho \frac{\partial}{\partial x_{j}}\left(\bar{U}_{j} k\right)=\frac{\partial}{\partial x_{j}}\left(\frac{\mu_{t}}{\sigma_{k}} \frac{\partial k}{\partial x_{j}}\right)-\rho \overline{u_{i}^{\prime} u_{j}^{\prime}} \frac{\partial \bar{U}_{i}}{\partial x_{j}}-\rho \varepsilon
$$

The energy dissipation rate

$$
\rho \frac{\partial}{\partial x_{j}}\left(\bar{U}_{j} \varepsilon\right)=\frac{\partial}{\partial x_{j}}\left(\frac{\mu_{t}}{\sigma_{\varepsilon}} \frac{\partial \varepsilon}{\partial x_{j}}\right)+C e_{1} P_{k} \frac{\varepsilon}{k}-C e_{2} \rho \frac{\varepsilon^{2}}{k}
$$

The model constants used in the present study are given in Table 1.

The $k-\varepsilon$ model has been used with success in the calculation of various turbulent jets. However, in flows with significant streamline curvature, the isotropic eddy viscosity assumption may not be able to describe the turbulent diffusion effects adequately.

\subsection{The Second-Order Model}

The second turbulence model considered in this study is a Reynolds Stress Model (RSM). The Reynolds stress equation is:

$$
\rho \frac{\partial}{\partial x_{k}}\left(\bar{U}_{k} \overline{u_{i}^{\prime} u_{j}^{\prime}}\right)=P_{i j}+D_{i j}+\overline{p^{\prime}\left(\frac{\partial u_{i}^{\prime}}{\partial x_{j}}+\frac{\partial u_{j}^{\prime}}{\partial x_{i}}\right)}-\frac{2}{3} \rho \varepsilon \delta_{i j}
$$

The first term on the right hand side is the production term due to the mean strain:

$$
P_{i j}=-\rho \overline{u_{i}^{\prime} u_{k}^{\prime}} \frac{\partial \bar{U}_{j}}{\partial x_{k}}-\rho \overline{u_{j}^{\prime} u^{\prime}}{ }_{k} \frac{\partial \overline{U_{i}}}{\partial x_{k}}
$$

The diffusion term is modelled as:

$$
D_{i j}=C_{s} \rho \frac{\partial}{\partial x_{k}}\left(\rho \frac{k}{\varepsilon} \overline{u_{k}^{\prime} u_{l}^{\prime}} \frac{\partial\left(\overline{u_{j}^{\prime} u_{j}^{\prime}}\right)}{\partial x_{l}}\right)
$$

Table 1. Turbulence constants for the first order $k$ - $\varepsilon$ model, where the value of $C_{\mu}$ is adapted for the axisymmetric jet case.

\begin{tabular}{ccccc}
\hline$C \mu$ & $C_{e, 1}$ & $C_{e, 2}$ & $\sigma_{k}$ & $\sigma_{\varepsilon}$ \\
\hline 0.06 & 1.44 & 1.92 & 1.00 & 1.30 \\
\hline
\end{tabular}


The pressure-strain correlation is:

$$
\begin{gathered}
\overline{p^{\prime}\left(\frac{\partial u_{i}^{\prime}}{\partial x_{j}}+\frac{\partial u_{j}^{\prime}}{\partial x_{i}}\right)}=\phi_{i j}^{I}+\phi_{i j}^{I I} \\
\phi_{i j}^{I}=-C_{1} \rho \varepsilon\left(\frac{\overline{u_{j}^{\prime} u_{j}^{\prime}}}{k}-\frac{2}{3} \delta_{i j}\right): \text { The return to isotropy }
\end{gathered}
$$

term.

$$
\phi_{i j}^{I I}=-C_{2}\left(P_{i j}-\frac{2}{3} P_{k k} \delta_{i j}\right): \text { The rapid term. }
$$

The model constants used in the present study are given in Table 2.

\section{NUMERICAL APPROACH}

The computations for the governing equations can be made using a parabolic marching procedure if the radial pressure gradients are small and the axial diffusion is neglected. Such a situation occurs if velocities in the two streams are comparable. Assuming parabolic conditions, a numerical solver has been developed using finite volume of Patankar [18].

The computations are performed up to an axial distance of approximately $100 \mathrm{D}$ with an axial forward step size of 0.01 times the local jet half width $\Delta x=0.01 L_{u}$ and 80 grid points in the radial direction are used. The radial expansion $\Delta r=\Delta x \frac{d L_{u}}{d x}$ is so small that it does not affect the assumption of an orthogonal grid. This means that the grid expands in the radial direction following the jet expansion and this is sufficient to obtain a grid independent numerical solution.

No boundary conditions are prescribed due to the parabolic nature of the flow. The computation progresses from section to section, and its implementation requires only the profiles at the jet nozzle. The boundary conditions at the nozzle exit are those of a fully developed pipe flow [19]. The radial velocity is zero at the nozzle and in the ambient. All variables at the radial jet boundary are equal to those in the ambient. At the axis of symmetry, the radial velocity and the radial gradients of other variables are set to zero. For all calculations, a small co-flow velocity value is used. For the turbulence quantities this implies a value of zero or a negligible small value. The kinetic energy and the Reynolds stress profile are used to derive the energy dissipation through the following relationship:

Table 2. Turbulence constants in the second order model, where the value of $C_{1}$ is adapted for the axisymmetric jet case.

\begin{tabular}{ccc}
\hline$C_{1}$ & $C_{2}$ & $C_{\mathrm{s}}$ \\
\hline 2.3 & 0.6 & 0.22 \\
\hline
\end{tabular}

$$
\varepsilon=\frac{C_{\mu} k^{2}}{(-\overline{u v})}\left(\frac{\partial U}{\partial r}\right)
$$

\section{RESULTS AND DISCUSSION}

The influence of co-flow is investigated using the experiments data of Djeridane [16]. The jet is ejected from a round nozzle of an internal diameter $D$ of $26 \mathrm{~mm}$ with various co-flow to jet velocity ratios of $U_{c o} / U_{j}=0.0$ and 0.1 . The experimental details are given in Table 3. The far field behavior of the computed quantities such as the velocity decay constant, the turbulence intensity, the spreading rate and the turbulent flux are presented and discussed.

Figure 1 shows, the jet centreline axial mean velocity $\left(U_{j}-U_{c o}\right) /\left(\bar{U}_{c}-U_{c o}\right)$ with and without co-flow as a function of the normalized distance $x / D$. It is seen that the velocity is somewhat overpredicted with experiment values. The predicted results obtained, using the two turbulence models, agree quite well with the experimental data of Djeridane [16]. The major visible effect of the co-flow is the jet decay rate reduction, in comparison with the free jet case.

Therefore, the jet without co-flow, tends to mix more rapidly with the ambient air than the co-flowing jets. In the far region, a hyperbolic decrease of the mean velocity is observed. However, for $20<x / D<50$, the velocity decay constant $K_{u}$, is defined by the following,

$$
\frac{\left(U_{j}-U_{c o}\right)}{\left(\bar{U}_{c}-U_{c o}\right)}=K_{u} \frac{x}{D}
$$

Table 3. Properties of the investigated turbulent co-flowing jets.

\begin{tabular}{ccccc}
\hline Jet/co-flow & $U_{\text {jet }}$ & $U_{c o}$ & $S_{\rho}$ & $\operatorname{Re}_{j}$ \\
\hline Air/air & 12 & 0.00 & 1 & 21000 \\
Air/air & 12 & 1.20 & 1 & 21000 \\
\hline
\end{tabular}

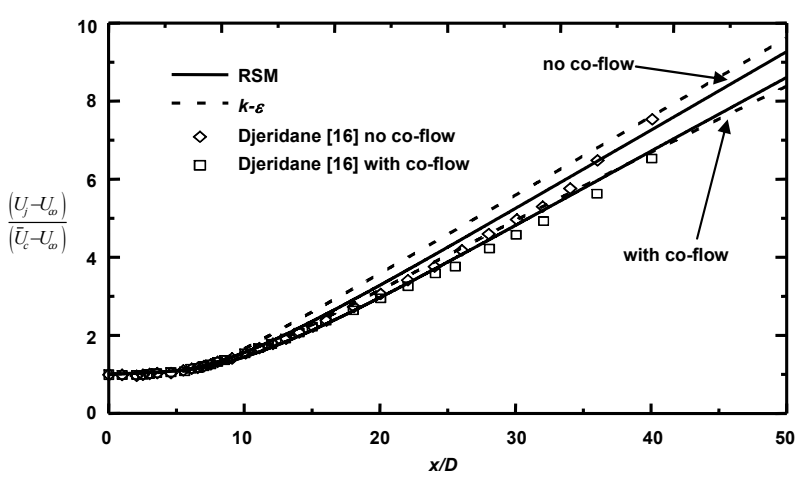

Figure 1. Centreline values of the axial mean velocity. 
This constant is closely related to the spreading rate, and the asymptotic value of the turbulence intensity.

The comparison of this decay constant with those found in the literature is a good validation tool for the developed computational code. The results of the present investigation give for the case with a co-flow, a value of $K_{u}=0.188$ with the second order model, and a value of $K_{u}=0.174$ with the first order model, while the experimental value is 0.176 Djeridane [16]. However, in the case of the jet without a co-flow, the value for $K_{u}$ is found to be 0.199 with the second model and 0.201 with the first order model. The experimental value of the slope $K_{u}$ is 0.218 Djeridane [16]. Table 4 recapitulates the asymptotic values of the mean velocity decay for various co-flow strengths of the different model predictions and compared to the experimental values of Djeridane [16].

Using the similarity law proposed by Chen and Rodi [20] $\left(U_{j} / \bar{U}_{c}=K_{u}\left(x / D_{e q}\right)\right)$, the influence of the velocity ratio on the decay $K_{u}$ is also described quite well by the most recent experimental study of Wang et al. [13] (shows $K_{u}=0.158$, with velocity ratios of $U_{c o} / U_{j}=0.075$ ) and Antoine et al. [11] (gives $K_{u}=0.146$, with velocity ratios of $U_{c o} U_{j}=0.05$ ). The major visible effect of the co-flow, when the velocity ratio increases, is the jet decay rate reduction.

Figure 2 features a comparison of the computational and experimental jet spreading rates of the velocity field based on the mean velocity half radius $L_{u}$. It is noticed that the half-width $L_{u}$ in the jets without a co-flow are much larger than those of the corresponding jets with a co-flow. Furthermore, with the first model, the predicted half-width of the jet obtained by the two jets cases agrees much better with the experimental data of Djeridane [16]. Here, the mean velocity half-width is defined by

Table 4. Comparison between the model predictions and measurements of the velocity flow field for asymptotic values of mean velocity decay, the spreading rate and the velocity fluctuation intensities at various co-flow strengths.

\begin{tabular}{ccccccc}
\hline \multirow{2}{*}{ Authors } & $U_{c o} / U_{j e t}$ & $S_{\rho}$ & $K_{u}$ & $S_{u}^{1 / 2}$ & $\frac{\sqrt{u_{c}^{2}}}{\left(\bar{U}_{c}-U_{c o}\right)}$ & $\frac{\sqrt{u_{\max }^{2}}}{\left(\bar{U}_{c}-U_{c o}\right)}$ \\
\hline RSM & 0.10 & 1.0 & 0.188 & 0.100 & 0.286 & 0.252 \\
RSM & 0.0 & 1.0 & 0.199 & 0.192 & 0.251 & 0.244 \\
$k-\varepsilon$ & 0.10 & 1.0 & 0.174 & 0.096 & 0.305 & 0.269 \\
$k-\varepsilon$ & 0.0 & 1.0 & 0.201 & 0.195 & 0.283 & 0.267 \\
Djeridane [16] & 0.10 & 1.0 & 0.176 & 0.132 & 0.261 & 0.219 \\
Djeridane [16] & 0.00 & 1.0 & 0.218 & 0.184 & 0.253 & - \\
Antoine et al. [11] & 0.075 & 1. & 0.146 & 0.128 & - & - \\
Wang et al. [13] & 0.05 & 1.0 & 0.158 & 0.12 & $0.25-0.30$ & - \\
\hline
\end{tabular}

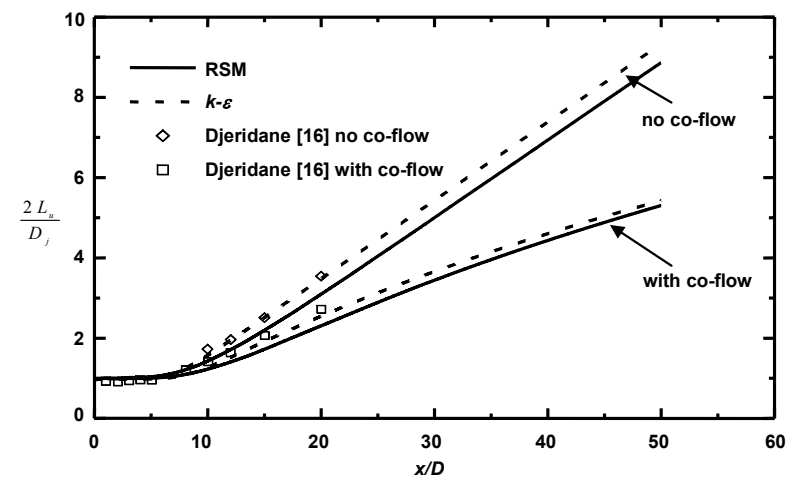

Figure 2. Centreline values of the mean velocity halfwidth.

$$
\frac{2 L_{u}}{D}=S_{u}^{1 / 2} \frac{x}{D}
$$

where $S_{u}^{1 / 2}$ is the mean velocity spreading rate. In the absence of a co-flow, both models predict a velocity spreading rate $S_{u}^{1 / 2}=0.192$ with the second model and $S_{u}^{1 / 2}=0.195$ with the first model. These rates are close to the experimental value $S_{u}^{1 / 2}=0.184$ of Djeridane [16]. In presence of a co-flow, the half-width is no longer a linear function of $x$, so $S_{u}^{1 / 2}$ can not be easily determined. Therefore $S_{u}^{1 / 2}$ depends on the axial distance and is not a useful concept in a co-flowing jet. However, the spreading rates $S_{u}^{1 / 2}=0.10$ and $S_{u}^{1 / 2}=0.096$, obtained by the two models with a co-flow are about $22 \%$ smaller than that the average experimental values of Djeridane [16] $S_{u}^{1 / 2}=0.132$, the air-air jet of Wang et al. [13] $S_{u}^{1 / 2}=0.12$, and the water-air jet of Antoine et al. [11] $S_{u}^{1 / 2}=0.128$. This low value of the velocity spreading rate can be attributed to the presence of the higher co-flowing stream and to the side walls position of the enclosure, compared to those of the latter authors, tending to reduce the jet expansion.

Figure 3 shows the axial profiles of the velocity fluctuation intensities $\sqrt{u_{c}^{2}} /\left(\bar{U}_{c}-U_{c o}\right)$ on the jet centerline with and without a co-flow. The predicted results obtained by the two turbulence models agree very well with the experimental data of Djeridane [16], and especially with the second order model. The asymptotic value of $\sqrt{u_{c}^{2}} /\left(\bar{U}_{c}-U_{c o}\right)$ is apparently strongly influenced by the used turbulence model. It is seen that the velocity fluctuation intensity with and without a co-flow is slightly overpredicted by the second order model, while for the first order model, the velocity fluctuation intensity is highly overpredicted. This is obvious since the $k-\varepsilon$ model is an isotropic model which then overestimates the velocity fluctuation intensity. Gharbi et al. [21] and Sanders et al. [22] have observed this same behaviour and concluded that this deviation is not due to the fact that the $k-\varepsilon$ model gives unsatisfactory results, rather it is the anisotropy that is badly predicted. 
Figure 3 also features a tendency toward a constant velocity fluctuation intensity value of 0.286 at $\mathrm{x} / \mathrm{D}>20$ with the second order model, and a value of 0.305 with the first order model, which are both close to the value 0.261 obtained by Djeridane [16]. Additionally, it is noticed at $\mathrm{x} / \mathrm{D}<20$ that, without a co-flow, the velocity turbulence intensity increases faster with $\mathrm{x} / \mathrm{D}$ than for the co-flow case. It is interesting to note that the predicted velocity fluctuation intensity shows an approximate asymptotic behaviour which increases in value with the increasing co-flow velocity.

Figure 4 shows the radial profiles of the mean velocity for the downstream section $x / D=20$, situated in the affinity region of the jet. It is noticed that both models agree reasonably well with the experimental data of Djeridane [16] for the co-flow case. Figure 5 presents the radial velocity fluctuation intensities profiles at $x / D$ $=20$. Qualitative agreement is obtained in the sense that both models predict a local maximum which is also observed experimentally. It should be mentioned again that the observed difference, between the experimental and the numerical values on the jet axis, is due to the chosen initial conditions. These values are of the order of $15 \%$. The axial mean velocity should decrease faster, and thus

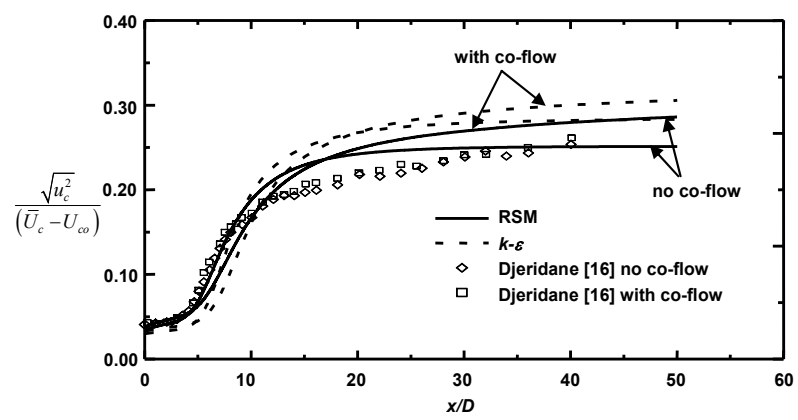

Figure 3. Centreline values of the velocity fluctuation intensities.

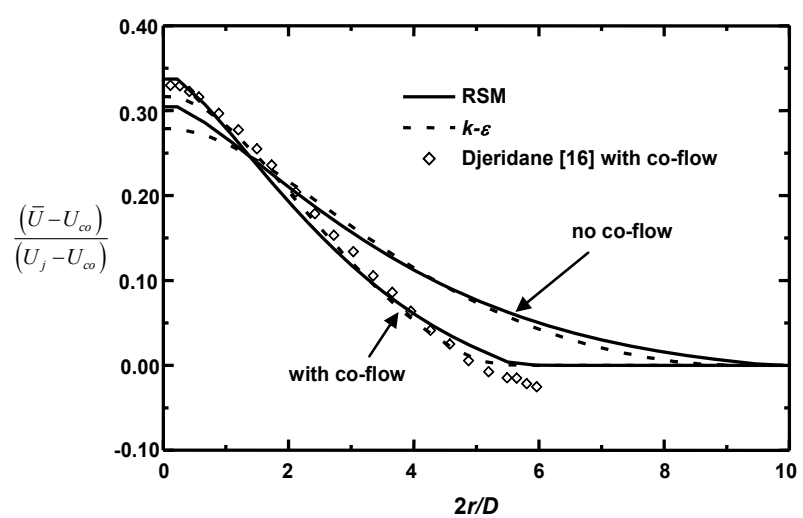

Figure 4. Radial profile of the normalized velocity fluctuation intensities at $x / D=20$.

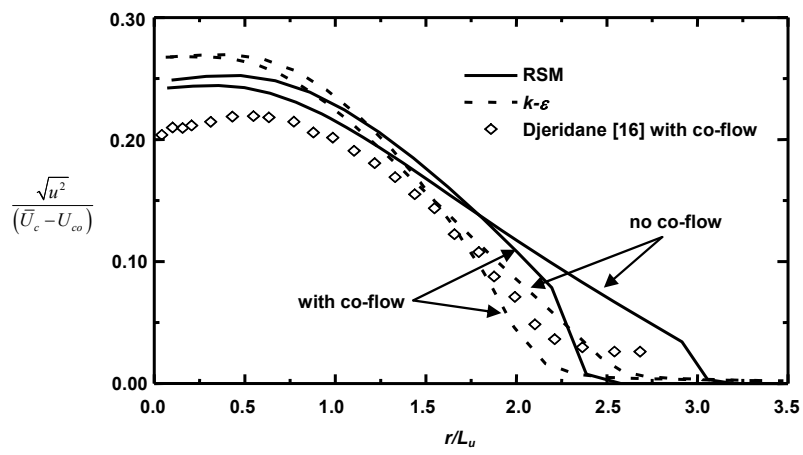

Figure 5. Radial profile of the mean velocity at $x / D=20$.

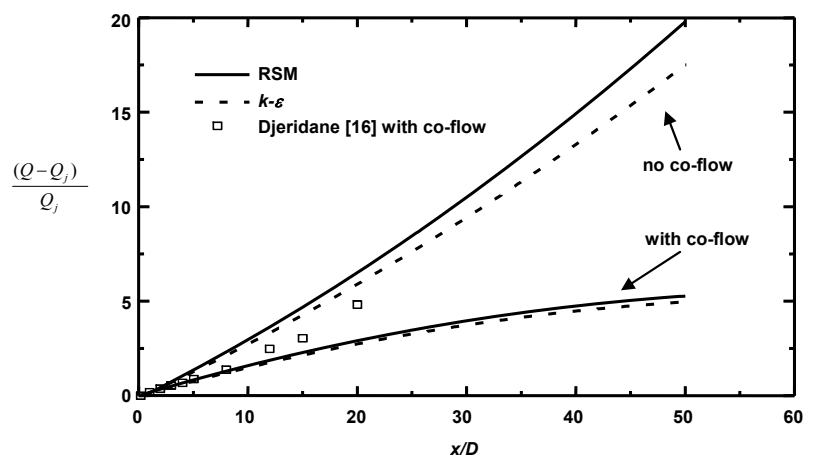

Figure 6. Evolution of the axial entrainment of air.

more efficient turbulent mixing is required. Furthermore, it should be noticed that the jet radial expansion is reduced when the co-flow is present.

The amount of air entrainment by the jet is determined by the time-average radial profiles of velocity. It relates the mass flow rate of the surrounding fluid entrained into the jet to the characteristic velocity difference between the jet and the co-flow.

$$
Q=2 \pi \int_{0}^{r(U=U c o)} \rho\left(\bar{U}-U_{c o}\right) r d r
$$

Based on this last definition, Figure 6 shows the axial evolution of the air entrainment. It is obvious that the coflow decreases considerably the air entrainment. A qualitative analysis would suggest that a co-flowing stream would restrict the radial in flow of air into the jet. Moreover, the free jets entrain from 30 to $75 \%$ more air than the co-flowing jets at any given axial location.

\section{CONCLUSIONS}

A turbulent jet with and without a co-flowing air has been theoretically and numerically investigated, using the first and the second order turbulence closure models. The calculation results show that both models qualitatively predict the behavior of jets with or without co-flowing air. An investigation of the asymptotic values for 
the mean velocity decay constant $K_{u}$, the spreading rate $S_{u}^{1 / 2}$ and the centerline value of the velocity fluctuation intensities has been presented. The predictions agree reasonably well with the very recent experimental study in the literature for axisymmetric jets. The major visible effect of the co-flow is the jet decay rate reduction, in comparison with the free jet case. However, based on entrainment definition, it is mainly shown that the coflow reduce the air entrainment.

\section{REFERENCES}

[1] Panchapakesan, N.R. and Lumley, J.L. (1993) Turbulence measurements in axisymmetric jets of air and helium, Part 2. Helium jet. Journal of Fluid Mechanics, 246, 225-247.

[2] Ruffin, E., Schiestel, E., Anselmet, F., Amielh, M. and Fulachier, L. (1994) Investigation of characteristic scales in variable density turbulent jets using a second-order model. Physics of Fluids, 6(8), 2785-2799.

[3] Chassaing, P., Harran, G. and Joly, L. (1994) Density fluctuation correlations in free turbulent binary mixing. Journal of Fluid Mechanics, 279, 239-278.

[4] Lucas, J.F. (1998) Analyse du champ scalaire au sein d'un jet turbulent axisymétrique à densité variable. Ph.D. Thesis, Université d'Aix-Marseille II, Marseille.

[5] Gazzah, M.H., Sassi, M., Sarh, B. and Gökalp, I. (2002) Simulation numérique des jets turbulent subsoniques à masse volumique variable par le modèle $k-\varepsilon$. International Journal of Thermal Sciences, 41, 51-62.

[6] Imine, B., Saber-Bendhina, A., Imine, O. and Gazzah, M.H. (2005) Effects of a directed co-flow on a non-reactive turbulent jet with variable density. International Journal of Heat and Mass Transfer, 42(1), 39-50.

[7] Gazzah, M.H., Belmabrouk, H. and Sassi, M. (2004) A numerical study of the scalar field in turbulent round jet with co-flowing stream. Computational Mechanics, 34(5), 430-437.

[8] Gazzah, M.H., Belmabrouk, H. and Sassi, M. (2005) Scalar transport modelling in turbulent round jets with co-flowing stream. International Journal of Thermal Sciences, 44(8), 766-773.

[9] Borean, J.L., Huilier, D. and Burnage, H. (1998) On the effect of a co-flowing stream on the structure of an axi- symmetric turbulent jet. Experimental Thermal and Fluid Science, 17(1-2), 10-17.

[10] Schefer, R.W. and Dibble, R.W. (2001) Mixture fraction field in a turbulent non-reacting propane jet. American Institute of Aeronautics and Astronautics Journal, 39(1), 64-72.

[11] Antoine, Y., Lemoine, F. and Lebouché, M. (2001) Turbulent transport of a passive scalar in a round jet discharging into a co-flowing stream. European Journal of Mechanics - B/Fluids, 20(2), 275-301.

[12] Hakem, M., Hazzab, A. and Ghenaim, A. (2007) Experimental investigation of elliptical jet in coflow. International Journal of Applied Engineering Research, 2(1), 31-43.

[13] Wang, P., Fröhlicha, J., Michelassib, V. and Rodi, W. (2008) Large-eddy simulation of variable-density turbulent axisymmetric jets. International Journal of Heat and Fluid Flow, 29(3), 654-664.

[14] Curtet, R. (1957) Contribution à l'étude théorique des jets de revolution. Extrait des Comptes rendus de l'Académie des Sciences, 244, 1450-1453.

[15] Steward, F.R. and Gurus, A.G. (1977) Aerodynamic of a confined jet with variable density. Combustion Science and Technology, 16(1-2), 29-45.

[16] Djeridane, T. (1994) Contribution à l'étude expérimentale de jets turbulents axisymétriques à densité variable. Ph.D. Thesis, Université d'Aix-Marseille II, Marseille.

[17] Schiestel, R. (1993) Modélisation et simulation des écoulements turbulents. Hermès Group, Paris.

[18] Patankar, S.V. (1980) Numerical heat transfer and fluid flow. Hemisphere Publishing, Washington, D.C.

[19] Laufer, J. (1953) The structure of turbulence in fully developed pipe flow. National Advisory Committee for Aeronautics-Report-1174, 417-434.

[20] Chen, C.J. and Rodi, W. (1980) Vertical turbulent buoyant jets - a review of experimental data. The Science and Application of Heat and Mass Transfer, Pergamon Press, New York.

[21] Gharbi, A., Ruffin, E., Anselmet, F. and Schiestel, R. (1996) Numerical modelling of variable density turbulent jets. International Journal of Heat and Mass Transfer, 39(9), 1865-1882.

[22] Sanders, J.P.H., Sarh, B. and Gökalp, I. (1997) Variable density effects in axisymmetric isothermal turbulent jets: a comparison between a first-and a second-order turbulence model. International Journal of Heat and Mass Transfer, 40(4), 823-842. 